\title{
The Global Burden of Pediatric Cryptosporidium Infections
}

\author{
Poonum S. Korpe ${ }^{1} \cdot$ Luther Bartelt $^{2}$
}

Published online: 16 July 2015

(C) Springer International Publishing AG 2015

\begin{abstract}
Cryptosporidiosis has been identified as a leading cause of diarrhea in young children worldwide. Infection results in significant short-term morbidity as well as long-term sequelae. Recent advancements in molecular diagnostics used in large multicenter prospective studies have led to the discovery that burden of Cryptosporidium disease is higher than previously recognized; however, the implications of this discovery are not fully understood. Epidemiologic studies suggest infection impacts long-term growth; however, the mechanisms driving this vicious cycle have yet to be elucidated, and it remains to be seen why malnutrition renders such high susceptibility to Cryptosporidium. Whole-genome sequencing has refined classification of anthroponotic and zoonotic strains and may shed light on novel modes of transmission and species -specific pathogenicity. As Cryptosporidium has been recognized as a significant pathogen with implications on child health it challenges us to determine the mechanisms of pathogenesis for this difficult to study parasite and also enforces the need to continue advancing innovative technologies in resource-limited settings to curb the impact of this neglected tropical disease.
\end{abstract}

'iThenticate: $4 \%$ - ok

This article is part of the Topical Collection on Protozoa

Poonum S. Korpe

pkorpe1@jhu.edu

Luther Bartelt

Lab2za@virginia.edu

1 3Division of Epidemiology, Johns Hopkins School of Public Health, 615 North Wolfe St, Baltimore, MD 21205, USA

2 Division of Infectious Diseases and International Health, University of Virginia, Charlottesville, VA 22903, USA
Keywords Cryptosporidium species · Cryptosporidium hominis · Cryptosporidium parvum · Child morbidity . Diarrhea $\cdot$ Malnutrition

\section{Introduction}

The apicomplexan protozoan Cryptosporidium spp. is the agent of cryptosporidiosis, best known for causing diarrhea in malnourished children and individuals living with HIV/AIDS. Due to the parasite's widespread global distribution, environmental hardiness with zoonotic potential, high rates of waterborne transmission in low resource settings, insufficient diagnostics, and lack of highly effective targeted therapy or vaccine, Cryptosporidium joined the list of neglected tropical diseases in 2006 . Advancements in diagnostics since that time have revealed that the burden of Cryptosporidium is greater than previously recognized, including new associations attributing Cryptosporidium as a major cause of moderate to severe pediatric diarrhea, mortality, and chronic long-term sequelae. While advancements have been made in the study of Cryptosporidium biology, pathogenesis, immune response, and transmission, several limitations including a paucity of experimental models continue to limit understanding of the parasite [1]. In this edition, we will review updates in our understanding of the impact of Cryptosporidium on children and then address important advancements and technologies, remaining knowledge gaps, and strategies towards achieving the goal of reducing the burden of Cryptosporidium.

\section{Endemic and Resource-Limited Settings/Morbidity and Mortality}

While advances have been made in reducing the mortality related to infectious diarrhea, largely due to improved efforts 
of symptomatic therapy with oral rehydrating solutions and intensified community-based care, the frequency of enteric pathogen exposure and long-term morbidities related to these infections remains high. In 2008, the United Nations estimated 4.4 million deaths in children under age five, with diarrheal disease and pneumonia as the leading causes of death [2]. Furthermore, diarrheal disease in early childhood has been associated with delayed age of starting school, cognitive impairment, decreased work productivity, and growth short falls [3-6].

It is now understood that the etiology of diarrhea in children living in low-income countries can be attributed to multiple enteropathogens due to lack of adequate sewage systems, overcrowding, and lack of access to potable water. In a prospective analysis examining both diarrheal and non-diarrheal stool specimens from children in Bangladesh, Taniuchi et al. recently identified not only diverse sequential enteropathogen exposures but the presence of greater than three different infectious agents per diarrheal episode [7]. There is growing evidence that certain pathogens are responsible for the greatest burden of diarrheal disease. Cryptosporidium is now recognized as one of these major diarrheal-associated pathogens across multiple recent studies [8••, 9, 10].

Many recent studies from South Asia and Africa have identified high rates of cryptosporidiosis in young children [11•, 12]. In India, it is estimated that cryptosporidiosis is responsible for up to 249,000 hospitalizations and 14,600 deaths per year in children under the age of two [11•]. The largest prospective study of pediatric morbidity and mortality from diarrhea to date, the Global Enteric Multicenter Study (GEMS), a case-control study over the first 3 years of life across seven different sites, with almost 500,000 child-years of observation, sought to identify leading etiologies of diarrhea in young children. GEMS demonstrated that Cryptosporidium species were responsible for the highest burden of diarrhea, second only to rotavirus. Furthermore, despite implementation of World Health Organization recommended management of diarrheal disease, Cryptosporidium was among the leading pathogens associated with prolonged symptoms and stood alone with increased mortality risk in children ages 12-23 months [8••].

In children, infection with Cryptosporidium spp. has been associated with prolonged diarrheal episodes lasting longer than 7 days [13-15]. Perhaps, even more important, earlier studies from Peru and Brazil suggest association of infection with long-term sequelae on child growth and development. In Peru, children with Cryptosporidium infection had persistent growth short falls 3-6 months after infection [16]. In Brazil, Guerrant et al. reported that Cryptosporidium infection during the first 2 years of life was significantly associated with decreased physical fitness 4-7 years later [4]. More recently, a larger cohort study from
Bangladesh has described a significant association of infection in the first 2 years of life and stunted growth at age two (Korpe et al., unpublished).

Beyond these epidemiologic associations, we are lacking in studies in humans that might shed light on the mechanism between infection and malnutrition. In vitro and animal studies suggest a role between infection and inflammation causing deranged immune signaling leading to enteropathy and poor growth [17-19]. These models, which need further validation in human studies, demonstrate that specific nutrient deprivation has a profound impact on the character and intensity of inflammation [19, 20], such as an apparent bias away from Th1-type primary immune responses (Bartelt, unpublished) similar to what has been shown in malnourished children in Haiti with cryptosporidiosis [21]. Also, the field observation that the APOE4/4 allelotype was protective against early childhood diarrhea and the cognitive impacts in malnourished children [22] was also shown to protect malnourished transgenic mice from weight loss and parasite burden following Cryptosporidium parvum challenge [23].

The impact of cryptosporidiosis may be much larger than what is apparent by measuring diarrheal burden alone. In a study of almost 400 children in Bangladesh, over $70 \%$ of children had at least one non-diarrheal infection with Cryptosporidium during the first 2 years of life [24]. In Southern India, a study of 186 young children documented asymptomatic infection in $60 \%$ [25]. And asymptomatic Cryptosporidium infection has also been documented at high rates in adults in endemic regions [26].

With the advent of molecular diagnostics, we are better able to detect enteric pathogens in stool and, in particular, describe otherwise difficult to detect organisms like enteric protozoa with far greater granularity than ever before [27]. While quantitative molecular diagnostics can associate parasite burden with severity of diarrhea during cryptosporidiosis [28••], what is the significance of low burden detection in asymptomatic children living in endemic countries? Is this simply a symptom of highly sensitive diagnostic techniques? Increasingly, there are findings to suggest when it comes to Cryptosporidium infection in young children; even nondiarrheal detections cannot be ignored. Few studies have suggested a relationship between non-diarrheal infection and poor growth [16]. In Bangladesh, children with non-diarrheal infection have twice the risk of becoming stunted compare to children without infection (Korpe, Duggal, Petri, unpublished). The impact of non-diarrheal infection on a child's growth and developmental potential need further study, since the burden of non-diarrheal infection in children in lowincome countries is so great. Additionally, the asymptomatic carriage of Cryptosporidium may provide a greater reservoir for spread of infection. 
Table 1 Most notable insights into Cryptosporidium

\begin{tabular}{ll}
\hline & Reference \\
\hline $\begin{array}{l}\text { Cryptosporidiosis is a leading cause of morbidity and } \\
\text { mortality from diarrhea in children under five. }\end{array}$ & {$[8 \cdot \bullet]$} \\
$\begin{array}{l}\text { Country-wide estimate of Cryptosporidium burden shows } \\
\text { that Cryptosporidium spp. is responsible for almost 15,000 }\end{array}$ & {$[11 \cdot]$} \\
$\quad \begin{array}{l}\text { deaths per year in children under two in India. } \\
\text { Advanced molecular diagnostics demonstrate that burden of }\end{array}$ & {$[28 \cdot \bullet]$} \\
$\quad \begin{array}{l}\text { parasite correlates with phenotype of disease. } \\
\text { Genotyping of Cryptosporidium spp. identifies unique risk } \\
\text { factors for infection. }\end{array}$ & {$[40 \cdot 41 \cdot]$}
\end{tabular}

\section{Transmission}

In addition to significant morbidity due to Cryptosporidium infection, children likely play an important role in the transmission cycle. Cryptosporidium is transmitted by fecal-oral spread and poses a challenge to public health systems due to resistance to chlorination and low infective dose. Sporadic outbreaks have been associated with contaminated water supplies [29] and notoriously in swimming pools [30]. However, in impoverished settings, direct anthroponotic or zoonotic spread may play a larger role than waterborne disease. A study from India demonstrated that households given bottled drinking water had just as high rates of cryptosporidiosis as households that used the municipal water supply, indicating that water source made no difference in risk of infection [26].

Using a serum IgG ELISA to the Cryptosporidium gp15 protein, it was determined that most children in South India experience their first exposure between 3 and 9 months of age, presumably prior to the introduction of non-breast milk supplementary food and water [31]. A 1994 study in Brazil identified a secondary attack rate of $39 \%$ in households with a child infected with Cryptosporidium. However, only half of these secondary cases manifested with diarrhea; the others had asymptomatic carriage [32]. An outbreak in Norwegian school children demonstrated a secondary attack rate of $17 \%$ [33]. Additionally, living in a
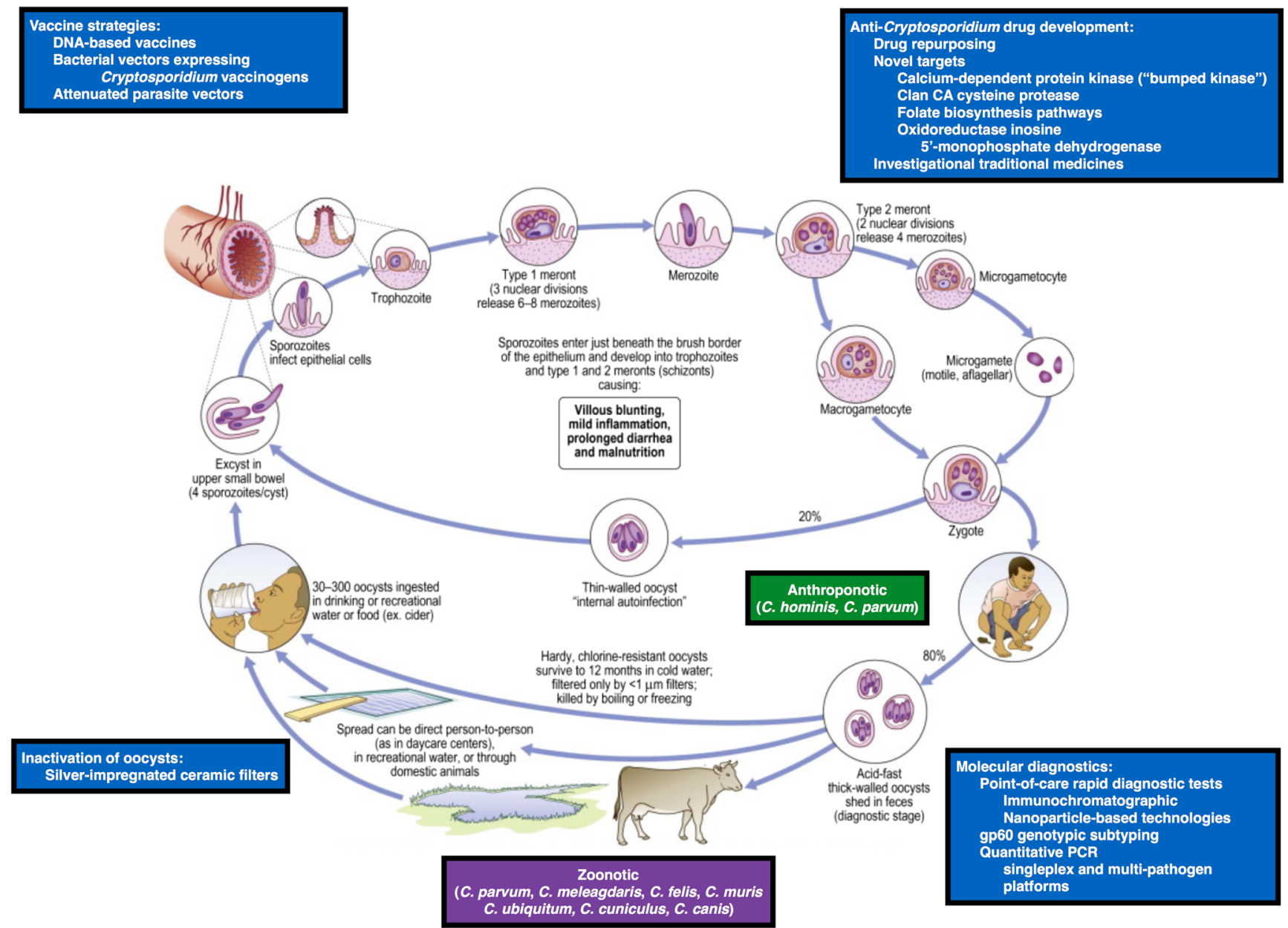

Fig. 1 The life cycle of Cryptosporidium and examples of investigational approaches to treat and control disease transmission. Adapted from "Cryptosporidiosis" Lima, Samie, and Guerrant in Tropical Infectious
Diseases: Principles, Pathogens, and Practice, 3rd Edition, Guerrant, Walker, Weller editors 
household with young children has consistently been identified as a risk factor for infection $[11 \bullet, 34]$. Thus, person-to-person spread likely plays an important role for spread of disease among children.

Furthermore, genotypic analyses demonstrate that despite close contact with domestic animals, anthroponotic spread may play a larger role in transmission, even among communities involved in animal husbandry [35-38].

In addition to fecal-oral spread, there is now emerging evidence that Cryptosporidium spp. may be a respiratory pathogen. Among children presenting with diarrhea to an acute care center in Uganda, 1/3 of children with Cryptosporidium infection also had sputum positive for Cryptosporidium by PCR [39•]. In Bangladesh, children presenting with Cryptosporidium diarrhea were over 2 times more likely to have concurrent pneumonia compared to children with noncryptosporidium associated diarrhea (adjusted Odds Ratio 2.7, CI 1.04, 7.2, $p=0.04$ )[Leung DT, Das SK, Malek MA et al., in press]. Therefore, respiratory spread may be an overlooked source of Cryptosporidium transmission.

\section{Strain and Species Variability}

To date, over 26 species in the genus Cryptosporidium have been described, with even further diversity within species. Advanced genotyping techniques are improving our understanding of the global distribution of specific subtypes (see Table 1 in accompanying article by Samie et al.) and may provide insight into species specific pathogenicity. For example, C. parvum has been associated with zoonotic transmission, but Widmer et al. applied whole-genome sequencing to determine that C. parvum isolate TU114 has more similarity to the anthroponotic Cryptosporidium hominis, explaining differences in this isolate's host range [40•]. In addition, molecular work has helped to better define risk factors for infection [42]. Chalmers et al. genotyped over 8000 Cryptosporidium isolates and found that a majority were $C$. parvum or C. hominis, and less than $1 \%$ were six other species. In this study, C. parvum infection was associated with younger age, and $C$. hominis was associated with recent travel [42]. Molecular techniques have also paved the way for identification of other species that infect humans, such as the cervine genotype, Cryptosporidium ubiquitum [41•].

Furthermore, we now see differences in geographic distribution of species and genotypes by subtyping of gp60 polymorphisms. In Spain, C. hominis 1bA10G2 was the predominant subtype identified [43]. In contrast, a long-term study of Bangladeshi children identified $C$. hominis subtypes Ie and $I d$ in greater than $90 \%$ of samples tested [Gilchrist, Petri, personal communication]. Further studies are needed to determine how subtype predicts pathogenicity and whether crossimmunity exists between subtypes.

\section{Conclusion}

The important impact of Cryptosporidium on the health of children living in under-resourced settings continues to be reinforced and refined [1, 44]. The burden of Cryptosporidium challenges our current best advances in supportive care for diarrheal diseases, pathogen detection in human stools and environmental samples, transmission of waterborne and fecal-oral enterics, and understandings of immunity to pathogens in intestinal mucosal compartment. This edition addresses examples of advances and strategies for responding to this heavy burden of Cryptosporidium through the application of improved and accessible point-of-care diagnostics for both clinical means and water quality testing measures, interrupting the Cryptosporidium transmission cycle, targeted anti-cryptosporidium drug development, and novel approaches to vaccine development (Fig. 1).

\section{Compliance with Ethics Guidelines}

Conflict of Interest The authors declare that they have no competing interests.

Human and Animal Rights and Informed Consent With regard to the authors' research cited in this paper, all procedures were followed in accordance with the ethical standards of the responsible committee on human experimentation and with the Helsinki Declaration of 1975, as revised in 2000 and 2008. With regard to the authors' animal research, all institutional and national guidelines for the care and use of laboratory animals were followed.

\section{References}

Papers of particular interest, published recently, have been highlighted as:

- Of importance

•- Of major importance

1. Checkley W, White AC, Jaganath D, Arrowood MJ, Chalmers RM, Chen XM, et al. A review of the global burden, novel diagnostics, therapeutics, and vaccine targets for cryptosporidium. Lancet Infect Dis. 2015;15(1):85-94.

2. United Nations Children's Fund WHO, The World Bank. Joint child malnutrition estimates - Levels and trends. WHO. 2012.

3. Lorntz B, Soares AM, Moore SR, Pinkerton R, Gansneder B, Bovbjerg VE, et al. Early childhood diarrhea predicts impaired school performance. Pediatr Infect Dis J. 2006;25(6):513-20.

4. Guerrant DI, Moore SR, Lima AA, Patrick PD, Schorling JB, Guerrant RL. Association of early childhood diarrhea and cryptosporidiosis with impaired physical fitness and cognitive function four-seven years later in a poor urban community in northeast Brazil. Am J Trop Med Hyg. 1999;61(5):707-13.

5. Niehaus MD, Moore SR, Patrick PD, Derr LL, Lorntz B, Lima AA, et al. Early childhood diarrhea is associated with diminished cognitive function 4 to 7 years later in children in a northeast Brazilian shantytown. Am J Trop Med Hyg. 2002;66(5):590-3. 
6. Moore SR, Lima AA, Conaway MR, Schorling JB, Soares AM, Guerrant RL. Early childhood diarrhoea and helminthiases associate with long-term linear growth faltering. Int J Epidemiol. 2001;30(6):1457-64.

7. Taniuchi M, Sobuz SU, Begum S, Platts-Mills JA, Liu J, Yang Z, et al. Etiology of diarrhea in Bangladeshi infants in the first year of life analyzed using molecular methods. J Infect Dis. 2013;208(11): 1794-802.

8.• Kotloff KL, Nataro JP, Blackwelder WC, Nasrin D, Farag TH, Panchalingam S, et al. Burden and aetiology of diarrhoeal disease in infants and young children in developing countries (the Global Enteric Multicenter Study, GEMS): a prospective, case-control study. Lancet. 2013;382(9888):209-22. Cryptosporidiosis is a leading cause of morbidity and mortality from diarrhea in children under five.

9. Mondal D, Minak J, Alam M, Liu Y, Dai J, Korpe P, et al. Contribution of enteric infection, altered intestinal barrier function, and maternal malnutrition to infant malnutrition in Bangladesh. Clin Infect Dis. 2012;54(2):185-92.

10. Nhampossa T, Mandomando I, Acacio S, Quintó L, Vubil D, Ruiz $\mathrm{J}$, et al. Diarrheal disease in rural Mozambique: burden, risk factors and etiology of diarrheal disease among children aged 0-59 months seeking care at health facilities. PLoS One. 2015;10(5):e0119824.

11. Sarkar R, Tate JE, Ajjampur SS, Kattula D, John J, Ward HD, et al. Burden of diarrhea, hospitalization and mortality due to cryptosporidial infections in Indian children. PLoS Negl Trop Dis. 2014;8(7):e3042. Country-wide estimate of Cryptosporidium burden shows that Cryptosporidium spp. is responsible for almost 15,000 deaths per year in children under two in India.

12. Eibach D, Krumkamp R, Al-Emran HM, Sarpong N, Hagen RM, Adu-Sarkodie Y, et al. Molecular characterization of Cryptosporidium spp. among children in rural Ghana. PLoS Negl Trop Dis. 2015;9(3):e0003551.

13. Lima AA, Moore SR, Barboza MS, Soares AM, Schleupner MA, Newman RD, et al. Persistent diarrhea signals a critical period of increased diarrhea burdens and nutritional shortfalls: a prospective cohort study among children in northeastern Brazil. J Infect Dis. 2000;181(5):1643-51.

14. Moore SR, Lima NL, Soares AM, Oriá RB, Pinkerton RC, Barrett $\mathrm{LJ}$, et al. Prolonged episodes of acute diarrhea reduce growth and increase risk of persistent diarrhea in children. Gastroenterology. 2010;139(4):1156-64

15. Khan WA, Rogers KA, Karim MM, Ahmed S, Hibberd PL, Calderwood SB, et al. Cryptosporidiosis among Bangladeshi children with diarrhea: a prospective, matched, case-control study of clinical features, epidemiology and systemic antibody responses. Am J Trop Med Hyg. 2004;71(4):412-9.

16. Checkley W, Gilman RH, Epstein LD, Suarez M, Diaz JF, Cabrera L, et al. Asymptomatic and symptomatic cryptosporidiosis: their acute effect on weight gain in Peruvian children. Am J Epidemiol. 1997;145(2):156-63.

17. Alcantara Warren C, Destura RV, Sevilleja JE, Barroso LF, Carvalho H, Barrett LJ, et al. Detection of epithelial-cell injury, and quantification of infection, in the HCT-8 organoid model of cryptosporidiosis. J Infect Dis. 2008;198(1):143-9.

18. Coutinho BP, Oriá RB, Vieira CM, Sevilleja JE, Warren CA, Maciel JG, et al. Cryptosporidium infection causes undernutrition, and conversely, weanling undernutrition intensifies infection. J Parasitol. 2008;94(6):1225-32.

19. Costa LB, JohnBull EA, Reeves JT, Sevilleja JE, Freire RS, Hoffman PS, et al. Cryptosporidium-malnutrition interactions: mucosal disruption, cytokines, and TLR signaling in a weaned murine model. J Parasitol. 2011;97(6):1113-20.

20. Castro IC, Oliveira BB, Slowikowski JJ, Coutinho BP, Siqueira FJ, Costa LB, et al. Arginine decreases Cryptosporidium parvum infection in undernourished suckling mice involving nitric oxide synthase and arginase. Nutrition. 2012;28(6):678-85.

21. Kirkpatrick BD, Daniels MM, Jean SS, Pape JW, Karp C, Littenberg B, et al. Cryptosporidiosis stimulates an inflammatory intestinal response in malnourished Haitian children. J Infect Dis. 2002;186(1):94-101.

22. Oriá RB, Patrick PD, Blackman JA, Lima AA, Guerrant RL. Role of apolipoprotein E4 in protecting children against early childhood diarrhea outcomes and implications for later development. Med Hypotheses. 2007;68(5):1099-107.

23. Azevedo OG, Bolick DT, Roche JK, Pinkerton RF, Lima AA, Vitek MP, et al. Apolipoprotein E plays a key role against cryptosporidial infection in transgenic undernourished mice. PLoS One. 2014;9(2): e89562.

24. Korpe PS, Liu Y, Siddique A, Kabir M, Ralston K, Ma JZ, et al. Breast milk parasite-specific antibodies and protection from amebiasis and cryptosporidiosis in Bangladeshi infants: a prospective cohort study. Clin Infect Dis. 2013;56(7):988-92.

25. Sarkar R, Ajjampur SS, Prabakaran AD, Geetha JC, Sowmyanarayanan TV, Kane A, et al. Cryptosporidiosis among children in an endemic semiurban community in southern India: does a protected drinking water source decrease infection? Clin Infect Dis. 2013;57(3):398-406.

26. Masarat S, Ahmad F, Chisti M, Hamid S, Sofi BA. Prevalence of Cryptosporidium species among HIV positive asymptomatic and symptomatic immigrant population in Kashmir. India Iran J Microbiol. 2012;4(1):35-9.

27. Platts-Mills JA, Liu J, Houpt ER. New concepts in diagnostics for infectious diarrhea. Mucosal Immunol. 2013;6(5):876-85.

28.• Liu J, Kabir F, Manneh J, Lertsethtakarn P, Begum S, Gratz J, et al. Development and assessment of molecular diagnostic tests for 15 enteropathogens causing childhood diarrhoea: a multicentre study. Lancet Infect Dis. 2014;14(8):716-24. Advanced molecular diagnostics demonstrate that burden of parasite correlates with phenotype of disease.

29. Fayer R. Cryptosporidium: a water-borne zoonotic parasite. Vet Parasitol. 2004;126(1-2):37-56.

30. Ng-Hublin JS, Hargrave D, Combs B, Ryan U. Investigation of a swimming pool-associated cryptosporidiosis outbreak in the Kimberley region of Western Australia. Epidemiol Infect. 2015;143(5):1037-41.

31. Sarkar R, Ajjampur SS, Muliyil J, Ward H, Naumova EN, Kang G. Serum IgG responses and seroconversion patterns to Cryptosporidium gp15 among children in a birth cohort in south India. Clin Vaccine Immunol. 2012;19(6):849-54.

32. Newman RD, Sears CL, Moore SR, Nataro JP, Wuhib T, Agnew DA, et al. Longitudinal study of Cryptosporidium infection in children in northeastern Brazil. J Infect Dis. 1999;180(1):167-75.

33. Johansen Ø, Hanevik K, Thrana F, Carlson A, Stachurska-Hagen T, Skaare D, et al. Symptomatic and asymptomatic secondary transmission of Cryptosporidium parvum following two related outbreaks in schoolchildren. Epidemiol Infect. 2015;143(8):1702-9.

34. Pereira MD, Atwill ER, Barbosa AP, Silva SA, García-Zapata MT. Intra-familial and extra-familial risk factors associated with Cryptosporidium parvum infection among children hospitalized for diarrhea in Goiânia, Goiás. Brazil Am J Trop Med Hyg. 2002;66(6):787-93.

35. Cruz JR, Cano F, Càceres P, Chew F, Pareja G. Infection and diarrhea caused by Cryptosporidium sp. among Guatemalan infants. J Clin Microbiol. 1988;26(1):88-91.

36. Molbak K, Hojlyng N, Gottschau A, Sa JC, Ingholt L, da Silva AP, et al. Cryptosporidiosis in infancy and childhood mortality in Guinea Bissau, West Africa. BMJ. 1993;307(6901):417-20.

37. Roy SL, DeLong SM, Stenzel SA, Shiferaw B, Roberts JM, Khalakdina A, et al. Risk factors for sporadic cryptosporidiosis 
among immunocompetent persons in the United States from 1999 to 2001. J Clin Microbiol. 2004;42(7):2944-51.

38. Ehsan AM, Geurden T, Casaert S, Parvin SM, Islam TM, Ahmed $\mathrm{UM}$, et al. Assessment of zoonotic transmission of Giardia and Cryptosporidium between cattle and humans in rural villages in Bangladesh. PLoS One. 2015;10(2):e0118239.

39. Mor SM, Tumwine JK, Ndeezi G, Srinivasan MG, KadduMulindwa DH, Tzipori S, et al. Respiratory cryptosporidiosis in HIV-seronegative children in Uganda: potential for respiratory transmission. Clin Infect Dis. 2010;50(10):1366-72.

40. Widmer G, Cacciò SM. A comparison of sequence and length polymorphism for genotyping Cryptosporidium isolates. Parasitology. 2015;142(8):1080-5. Genotyping of Cryptosporidium spp. identifies unique risk factors for infection.
41. Li N, Xiao L, Alderisio K, Elwin K, Cebelinski E, Chalmers R, et al. Subtyping Cryptosporidium ubiquitum, a zoonotic pathogen emerging in humans. Emerg Infect Dis. 2014;20(2):217-24. Genotyping of Cryptosporidium spp. identifies unique risk factors for infection.

42. Chalmers RM, Elwin K, Thomas AL, Guy EC, Mason B. Longterm Cryptosporidium typing reveals the aetiology and speciesspecific epidemiology of human cryptosporidiosis in England and Wales, 2000 to 2003. Euro Surveill. 2009. 14(2).

43. Segura R, Prim N, Montemayor M, Valls ME, Muñoz C. Predominant virulent IbA10G2 subtype of Cryptosporidium hominis in human isolates in Barcelona: a 5-year study. PLoS One. 2015;10(3): 0121753.

44. Savioli L, Smith H, Thompson A. Giardia and cryptosporidium join the 'neglected diseases initiative'. Trends Parasitol. 2006;22(5):203-8. 